\title{
MATHEMATICAL FORMALISM FOR ISOTHERMAL LINEAR IRREVERSIBILITY
}

\author{
Hong Qian \\ Department of Applied Mathematics, Universityi of Washington, \\ Seattle, WA 98195-2420 \\ E-mail: qian@amath.washington.edu
}

\begin{abstract}
We prove the equivalence among symmetricity, time reversibility, and zero entropy production of the stationary solutions of linear stochastic differential equations. A sufficient and necessary reversibility condition expressed in terms of the coefficients of the equations is given. The existence of a linear stationary irreversible process is established. Concerning reversibility, we show that there is a contradistinction between any 1-dimensional stationary Gaussian process and stationary Gaussian process of dimension $n>1$. A concrete criterion for differentiating stationarity and sweeping behavior is also obtained. The mathematical result is a natural generalization of Einstein's fluctuation-dissipation relation, and provides a rigorous basis for the isothermal irreversibility in a linear regime which is the basis for applying Onsager's theory to macromolecules in aqueous solution.
\end{abstract}

Key Words: Brownian motion, Entropy Production Rate, Fluctuation-Dissipation Relation, Reversibility, Stochastic Macromolecular Mechanics, Sweeping 


\section{INTRODUCTION}

The stochastic differential equation

$$
\frac{d x}{d t}=\boldsymbol{b}(x)+\Gamma \xi(t), \quad x \in \boldsymbol{R}^{n}
$$

with $\Gamma$ being a nonsingular matrix and $\xi(t)$ being the "derivative" of a $n$-dimensional Wiener process, has wide applications in science and engineering [9]. One standard method for attacking this equation is by finding the fundamental solution to its corresponding Fokker-Planck (Kolmogorov forward) equation

$$
\frac{\partial P}{\partial t}=\nabla \cdot\left(\frac{1}{2} A \nabla P-\boldsymbol{b}(x) P\right), \quad\left(A=\Gamma \Gamma^{T}\right)
$$

a parabolic equation for the transition probability $P\left(x, t \mid x^{0}\right)$ defined on the entire $\boldsymbol{R}^{n}$ with the integrability condition

$$
\int_{\boldsymbol{R}^{n}}|P(x, t)| d x<+\infty .
$$

That is $P \in L^{1}\left[\boldsymbol{R}^{n}\right]$. With this condition, it has been difficult to obtain detailed, rigorous understanding of the partial differential equation (PDE) in (2). However, for a class of PDE satisfying the regular conditions for the Cauchy problem, which include linear $\boldsymbol{b}(x)$, the existence and uniquess of a positive fundamental solution is guaranteed. Furthermore, the existence and uniqueness of the solution to the Cauchy problem with initial data $f(x)\left(|f(x)| \leq c e^{\alpha|x|^{2}}\right.$ with constant $c>0$ and $\left.\alpha>0\right)$ is proven. The above mentioned regular condition for Cauchy problem and its consequences can be found in [6]. In addition, a result on the nonexistence of a stationary solution in $L^{1}$, called sweeping, is also obtained. This is stated as follows [6]:

Assume that the coefficients of equation (2) are regular for the Cauchy problem. Further assume that all stationary nonnegative solution of (2) and (3) are of the form $c u(x)$ where $u(x)>0$ almost everywhere and $c$ is a nonnegative constant. Then the solution of (2) is either asymptotically stable or sweeping. Asymptotic stability occurs when

$$
I \equiv \int_{\boldsymbol{R}^{n}} u(x) d x<\infty
$$

and sweeping when $I=\infty$. 
The condition in this sweeping theorem is satisfied because of the uniqueness and positivity of the fundamental solution to (2) and the corresponding Cauchy problem [6]. No criterion has been given to assert when the sweeping or asymptotically stable behavior indeed occurr.

In this paper, we show that this problem can be completely solved for linear $\boldsymbol{b}(x)$. A sufficient and necessary condition for the sweeping behavior is obtained. Within the nonsweeping case, the stationary processes can be further classified as either reversible or irreversible. A series of sufficient and necessary conditions, and a criterion based on the coeffients of (1) are derived for this classification. The distributions concerned are all Gaussian. In the case of $n=1$, Gaussian stationary processes are necessarily reversible [24]. Part of our results, thus, may be regarded as a further development of this theorem for arbitrary $n>1$.

The stochastic differential equation given in (1) defines a Markovian process known as diffusion. This theory has been a well accepted mathematical model for the statistical behavior of molecular systems, following the work of Einstein, Ornstein and Uhlenbeck, Onsager, Keizer, and other physcists $[23,2,8,12,13]$. One of the central pieces in the physicists' work is the fluctuation-dissipation relation. Until now, however, the role of this deep relationship has not led to any mathematical conclusion in the theory of stochastic differential equation.

It turns out, the fluctuation-dissipation relation naturally emerges from the mathematical results of the present paper. We will show that the standard fluctuation-dissipation relation is a necessary but not a sufficient condition for time-reversibility. Our reversibility condition, on the other hand, can be viewed as a stronger form of fluctuation-dissipation relation which also has a closer resemblance to Einstein's original one. Time-reversibility is an important concept in which the physicists are interested. In connection to this end, we introduce the concept of entropy production rate, derive its analytical expression and prove that in a stationary process it is zero if and only if the process is reversible. Finally, we provide the condition under which a stationary irreversible Gaussian process exists.

\section{THERMODYNAMICS, HEAT DISSIPATION, AND ENTROPY PRODUCTION RATES}

In recent years, with the increasing number of applications of the stochastic model (1) or (2) to a wide range of macromolecular processes [4, 5, 14, 15], it has become evident that an axiomatic isothermal thermodynamic formalism can be established based on the stochastic differential equation. In this section, we give a brief introduction of this emerging theoretical framework we called stochastic macromolecular mechanics [15]. 
Following Lebowitz and Spohn [7], we introduce the instananeous heat dissipation functional $W_{t}[16]$ :

$$
d W_{t}=(A / 2)^{-1} \boldsymbol{b}\left(X_{t}\right) \circ d X_{t}=2 A^{-1} \boldsymbol{b}\left(X_{t}\right) \cdot d X_{t}+\nabla \cdot \boldsymbol{b}\left(X_{t}\right) d t
$$

where o denotes the integral in the Stratonovich sense. According to this definition, the heat dissipation, the left-hand-side of (4), is equal to the work done by the system, the right-hand-side of (4). The work is the product of force and displacement; the force is the product of the frictional coefficient $\left(2 A^{-1}\right)$ and velocity $\boldsymbol{b}(x)$. This is the law of energy conservation. It then follows that the mean rate of the heat dissipation

$$
\mathrm{hdr}=\frac{d}{d t} E\left[W_{t}\right]=\int_{\boldsymbol{R}^{n}} 2\left(A^{-1} \boldsymbol{b}(x)\right) \cdot \boldsymbol{J} d x
$$

in which

$$
\boldsymbol{J}=-\frac{1}{2} A \nabla P(x, t)+\boldsymbol{b}(x) P(x, t)
$$

denoting the probability flux in $(2): \partial P(x, t) / \partial t=-\nabla \cdot \boldsymbol{J}$, and the probability density $P(x, t)$ is the solution to $(2)$. In mechanical terms, the mean heat dissipation rate is the product of force $\left(2 A^{-1} \boldsymbol{b}(x)\right)$ and flux $(\boldsymbol{J})$ [8].

The thermodynamic force of Onsager is different from the mechanical force $2 A^{-1} \boldsymbol{b}(x)$. In terms of (2), the Onsager's thermodynamic force $\boldsymbol{\Pi}(x)$, called affinity by chemists, is [4]

$$
\begin{aligned}
\boldsymbol{\Pi}(x) \cdot d x & =\log \left(\frac{P(x+d x, t \mid x) P(x)}{P(x, t \mid x+d x) P(x+d x)}\right) \\
& \approx\left\{2 A^{-1} \boldsymbol{b}(x)-\nabla \log P(x)\right\} \cdot d x
\end{aligned}
$$

in which $\approx$ is valid for small $d x$. Therefore, the thermodynamic force $\boldsymbol{\Pi}$ and flux $\boldsymbol{J}$ are related by $\boldsymbol{J}=\frac{P}{2} A \boldsymbol{\Pi}$, in which the matrix $A$ is symmetric. This relation reflects the Onsager's reciprocal relations [10]. When applying (1) to molecular motors, a subspace of $x$ represents the internal motion of a macromolecule, and the remains of the $x$ represents the external movement of the entire macromolecule [11]. Hence the relation between $\boldsymbol{J}$ and $\Pi$ reflects a certain symmetry between the chemical flux, the mechanical movement, the chemical energy source, and the mechanical work in the energy convertion.

We now introduce another two important thermodynamic quantities: the entropy and entropy production rate (epr). The resulting analytical expression will then be used as a rigorous definition for the remains of the paper. 
We use the well-known definition for entropy

$$
e[P]=-\int_{\boldsymbol{R}^{n}} P(x, t) \log P(x, t) d x
$$

which is a functional of the probability density $P(x, t)$, the solution of $(2)$.

The rate of the increase of entropy is

$$
\begin{aligned}
\dot{e}[P] & =\int_{\boldsymbol{R}^{n}}(\log P+1) \nabla \cdot \boldsymbol{J} d x \\
& =-\int_{\boldsymbol{R}^{n}} P^{-1}\left(\nabla P-2 A^{-1} \boldsymbol{b}(x) P\right) \cdot \boldsymbol{J} d x-\int_{\boldsymbol{R}^{n}} 2 A^{-1} \boldsymbol{b}(x) \cdot \boldsymbol{J} d x \\
& =\int_{\boldsymbol{R}^{n}} \boldsymbol{\Pi} \cdot \boldsymbol{J} d x-\int_{\boldsymbol{R}^{n}} 2 A^{-1} \boldsymbol{b}(x) \cdot \boldsymbol{J} d x \\
& =\mathrm{epr}-\mathrm{hdr} .
\end{aligned}
$$

In the derivation, we used equation (2) and integration by parts, assumed no flux boundary condition and matrix $A$ being nonsingular. It is meaningful from the thermodynamic point of view to identify the first term in (8) with the entropy production rate which equals to the product of thermodynamic force and flux, and the second term is exactly the heat dissipation rate. In a time independent stationary state, the $\dot{e}=0$, and the entropy production is balanced by the heat dissipation. This is the case for an isothermal nonequilibrium steady-state.

For a single macromolecule immersed in a fluid with constant temperature, our epr in (8) is related to Onsager's dissipation function epr $/ 2=\Phi$, our $h d r$ is his $\dot{S}^{*}$, and our $\dot{e}$ is his $\dot{S}$. Therefore, (8) corresponds to $\dot{S}=2 \Phi-\dot{S}^{*}$ in which $2 \Phi$ is a "source" term, entropy production, $\dot{S}^{*}$ is the heat giving out by the system to the surrounding fluid, and $\dot{S}$ is the entropy change of the system proper.

We now define the entropy production rate and time reversibility.

Definition 2.1. The entropy production rate, epr, of a stationary diffusion process defined by (1) is

$$
\frac{1}{2} \int\left(\nabla \log P(x)-2 A^{-1} \boldsymbol{b}(x)\right)^{T} A\left(\nabla \log P(x)-2 A^{-1} \boldsymbol{b}(x)\right) P(x) d x .
$$

It is seen that the epr is always non-negative. This is the second law of thermodynamics. This paper is to establish a mathematical relationship between $e p r=0$ and the time reversibility defined as follows:

Definition 2.2. A stationary stochastic process $\{x(t) ; t \in \boldsymbol{R}\}$ is time reversible if for every positive integer $m$ and every $t_{1}, t_{2}, \ldots, t_{m} \in \boldsymbol{R}$, the joint 
probability distribution

$$
P\left(x\left(t_{1}\right), x\left(t_{2}\right), \ldots, x\left(t_{m}\right)\right)=P\left(x\left(-t_{1}\right), x\left(-t_{2}\right), \ldots, x\left(-t_{m}\right)\right) .
$$

Eq. 9 also indicates that the epr equals zero if and only if $2 A^{-1} \boldsymbol{b}=$ $\nabla \log P$. That is the force $2 A^{-1} \boldsymbol{b}$ being conservative with a potential: $2 A^{-1} \boldsymbol{b}=-\nabla U$. For systems satisfying the potential condition, the thermodynamic force $\boldsymbol{\Pi}$ also has a potential, $\boldsymbol{\Pi}=-\nabla(U(x)+\log P(x))$, whose expectation is precisely the Helmholtz free energy:

$$
\begin{aligned}
\Psi[P] & =E[U(x)+\log P(x)] \\
& =\int_{\boldsymbol{R}^{n}} P(x, t) U(x) d x-e[P] .
\end{aligned}
$$

in which the first term is the mean internal energy. It is easy to show that $\dot{\Psi}=-e p r \leq 0$ : the free energy increases and reaches its maximum at an equilibrium. It turns out that $\Psi$ is related to the relative entropy [17], and it is well-known that relative entropy is a Lyapunov function for Eq. 2 [6]. Finally, with the potential condition, the heat dissipation functional $d W_{t}$ $=d U\left(X_{t}\right)$, the internal energy fluctuation. Hence, $W_{t}$ is stationary and its expectation and variance are the internal energy and heat capacity of a single macromolecule at thermal equilibrium. A thermal equilibrium is necessarily time reversible.

\section{LINEAR THEORY OF REVERSIBLE STATIONARY PROCESSES}

Let's consider the linear stochastic differential equation of order $n$ :

$$
\frac{d x}{d t}=-B x+\Gamma \xi(t)
$$

where

$$
x(t)=\left[\begin{array}{c}
x_{1}(t) \\
\vdots \\
x_{n}(t)
\end{array}\right], \quad B=\left[\begin{array}{ccc}
b_{11} & \cdots & b_{1 n} \\
\vdots & & \vdots \\
b_{n 1} & \cdots & b_{n n}
\end{array}\right], \quad \Gamma=\left[\begin{array}{ccc}
\gamma_{11} & \cdots & \gamma_{1 n} \\
\vdots & & \vdots \\
\gamma_{n 1} & \cdots & \gamma_{n n}
\end{array}\right]
$$

is nonsingular, and

$$
\xi(t)=\left[\begin{array}{c}
\xi_{1}(t) \\
\vdots \\
\xi_{n}(t)
\end{array}\right]
$$


is the "white noise" vector which should be considered, from a mathematical point of view, as the derivative of a $n$-dimensional Wiener process. Formally, we have $E\left[\xi(t) \xi^{T}\left(t^{\prime}\right)\right]=\delta\left(t-t^{\prime}\right) I$ where $I$ is the identity matrix.

Heuristically speaking, near any stable fixed point of $\boldsymbol{b}(x)=0$ in a nonlinear (1) there is a linear approximation in the form of (11). Therefore, the mathematical analysis presented in the present paper can be considered as the linear theory near such fixed point for the nonlinear system. The linear approximation leads to a Gaussian process defined by (11).

The probability density of the solution of (11), $P(x, t)$, satisfies the Fokker-Planck equation

$$
\frac{\partial P}{\partial t}=\nabla \cdot\left(\frac{1}{2} A \nabla P+B x P\right)
$$

where $A=\Gamma \Gamma^{T}$, which is positive definite since we assume $\Gamma$ is nonsingular. Hence (14) is uniformly parabolic.

THEOREM 3.1. The following five statements about (11) are equivalent:

(i) Its $B$ and $\Gamma \Gamma^{T}=A$ satisfy a symmetry condition $A^{-1} B=\left(A^{-1} B\right)^{T}$ which is positive definite;

(ii) It has a stationary Gaussian solution with a symmetric two-time covariance matrix $R\left(t, t^{\prime}\right)=E\left[x(t) x^{T}\left(t^{\prime}\right)\right]$;

(iii) It defines a time reversible stationary process;

(iv) Its corresponding elliptic operator (the right-hand-side of (14)) is symmetric with respect to a positive function $w^{-1}(x), w(x) \in L^{1}$;

(v) Its stationary process has zero entropy production rate (epr).

Remark 1: According to [6], the stationary process mentioned above is unique.

Remark 2: The statement $(i)$ is also equivalent to $A^{-1} B x$ having a potential function $U(x)=\frac{1}{2} x^{T} A^{-1} B x$ and $\int_{\boldsymbol{R}^{n}} e^{-U(x)} d x<\infty$. The reversibility under this potential condition has been announced for the general nonlinear equation (1) but without proof [19, 11], and discussed in $[3,18]$ for the equation on compact manifold. In this paper, we provide a simple proof for the linear case.

Proof. (i) $\Rightarrow$ (ii). The symmetry and positive definiteness of $A^{-1} B=$ $\Gamma^{-T} \Gamma^{-1} B$ implies that $\Gamma^{-1} B \Gamma$ is also symmetric and positive definite. Hence there exists an orthogonal matrix $Q, Q^{T}=Q^{-1}$, such that $\Gamma^{-1} B \Gamma=$ $Q \Lambda Q^{-1}$ where $\Lambda$ is a diagonal matrix with all positive eigenvalues $\lambda_{1}, \lambda_{2}, \ldots, \lambda_{n}$. Thus

$$
B=\Gamma Q \Lambda Q^{-1} \Gamma^{-1}
$$


which has the same $\lambda$ 's as eigenvalues. Hence it is nonsingular. Substituting (15) into (11), we have

$$
\frac{d y}{d t}=-\Lambda y+\zeta(t)
$$

where $y=Q^{T} \Gamma^{-1} x, \zeta(t)=Q^{T} \xi(t)$, and $E\left[\zeta(t) \zeta^{T}\left(t^{\prime}\right)\right]=E\left[\xi(t) \xi^{T}\left(t^{\prime}\right)\right]=$ $\delta\left(t-t^{\prime}\right) I$. (16) is completely diagonalized. Its solution are $n$ OrnsteinUhlenbeck processes each with transition probability

$$
P\left(y_{i}, t \mid y_{i}^{0}\right)=\frac{1}{\sqrt{2 \pi} \omega_{i}(t)} \exp \left[-\frac{\left(y_{i}-y_{i}^{0} e^{-\lambda_{i} t}\right)^{2}}{2 \omega_{i}^{2}(t)}\right]
$$

and stationary distribution

$$
P\left(y_{i}\right)=\frac{1}{\sqrt{2 \pi} \omega_{i}(\infty)} \exp \left[-\frac{y_{i}^{2}}{2 \omega_{i}^{2}(\infty)}\right]
$$

where

$$
\omega_{i}^{2}(t)=\frac{1}{2 \lambda_{i}}\left(1-e^{-2 \lambda_{i} t}\right) .
$$

Furthermore, the two-time covariance matrix for the stationary process is

$$
E\left[y(t) y^{T}\left(t^{\prime}\right)\right]=\frac{1}{2} e^{-\Lambda\left|t-t^{\prime}\right|} \Lambda^{-1} .
$$

Therefore, by substituting $y=Q^{T} \Gamma^{-1} x$ back into the above equations, we have

$$
P\left(x, t \mid x^{0}\right)=\frac{\|E\|}{\sqrt{2 \pi}} \exp \left[-\frac{1}{2}\left(x-e^{-B t} x^{0}\right)^{T} E(t)\left(x-e^{-B t} x^{0}\right)\right]
$$

in which matrix

$$
E^{-1}(t)=\frac{1}{2}\left(I-e^{-2 B t}\right) B^{-1} \Gamma \Gamma^{T} .
$$

The stationary covariance $\Xi=E^{-1}(\infty)=\frac{1}{2} B^{-1} A$ gives a stationary Gaussian distribution. Combining with the transition probability in (20) and Markovian properties, a Gaussian stationary process with covariance $\Xi$ is obtained for (11).

Finally, substituting $y=Q^{T} \Gamma^{-1} x$ back into (19), the stationary two-time covariance matrix

$$
R\left(t, t^{\prime}\right)=E\left[x(t) x^{T}\left(t^{\prime}\right)\right]=\frac{1}{2} e^{-B\left|t-t^{\prime}\right|} B^{-1} A .
$$


We now show that $R\left(t, t^{\prime}\right)$ is symmetric. $A^{-1} B=B^{T} A^{-1}$ immediately leads to $B A=A B^{T}$, which gives:

$$
B^{n} A=B^{n-1} A B^{T}=B^{n-2} A\left(B^{2}\right)^{T}=\ldots=A\left(B^{n}\right)^{T} .
$$

That is $B^{n} A=\left(B^{n} A\right)^{T}$ for any interger $n$. Therefore matrix $R\left(t, t^{\prime}\right)$ is symmetric.

(ii) $\Rightarrow$ (iii). A Gaussian process $x(t)$ is completely determined by its expectation $E[x(t)]$ and covariance $E\left[x(t) x^{T}\left(t^{\prime}\right)\right]$. A stationary Markovian Gaussian process is completely determined by the joint distribution $P\left(x_{1}, x_{0}\right)$. The Gaussian $P\left(x_{1}, x_{0}\right)$ is determined by all its first and second moments, among which are the two-time covariance matrix $R(t, 0)=$ $E\left[x(t) x_{0}^{T}\right]$. Therefore, the symmetric matrix $R\left(t, t^{\prime}\right)=R\left(\left|t-t^{\prime}\right|, 0\right)$ leads to $P\left(x_{1}, x_{0}\right)=P\left(x_{0}, x_{1}\right)$; and with the Markovian property the stationary Gaussian process is then time reversible. ${ }^{1}$

(iii) $\Rightarrow$ (iv). For a stationary Markov process $x(t)$, the joint probability distribution $P\left(x\left(t_{1}\right), x\left(t_{2}\right), \ldots, x\left(t_{m}\right)\right.$ is uniquely determined by the stationary distribution $P_{e q}(x)$ and the transition probability $P(y, t \mid x)$. By the standard method in probability, the time reversibility implies [20]

$$
\begin{aligned}
& \int_{\boldsymbol{R}^{n}} \int_{\boldsymbol{R}^{n}} \phi(x) P(x, t \mid y) P_{e q}(y) \psi(y) d x d y \\
= & \int_{\boldsymbol{R}^{n}} \int_{\boldsymbol{R}^{n}} \psi(y) P(y, t \mid x) P_{e q}(x) \phi(x) d x d y
\end{aligned}
$$

being valid for arbitrary $\phi, \psi \in D\left[\boldsymbol{R}^{n}\right]$ where $D$ is the space of smooth functions with compact supports. Note that $P(y, t \mid x)$ is the fundamental solution to (14),

$$
\frac{d}{d t}\left(\int_{\boldsymbol{R}^{n}} P(y, t \mid x) f(x) d x\right)_{t=0}=\mathcal{L}[f(y)]
$$

where the differential operator

$$
\mathcal{L}[f(x)]=\nabla \cdot\left(\frac{1}{2} A \nabla f+B x f\right)
$$

\footnotetext{
${ }^{1}$ It is interesting to compare the cases of $n=1$ and $n>1$ : It is known in statistics literature [24] that any 1-dimensional stationary Gaussian process is necessarily time reversible, and any n-dimensional stationary Gaussian process can be realized as a solution of (11) [21]. Our result shows that for $n>1$ the reversibility does not generally hold.
} 
Differntiating both sides of (21) with respect to $t$ at $t=0$, we have

$$
\int_{\boldsymbol{R}^{n}} \phi(x) \mathcal{L}\left[P_{e q}(x) \psi(x)\right] d x=\int_{\boldsymbol{R}^{n}} \psi(y) \mathcal{L}\left[P_{e q}(y) \phi(y)\right] d y .
$$

let $f(x)=\phi(x) P_{e q}(x)$ and $g(x)=\psi(x) P_{e q}(x)$, then $f$ and $g$ are two arbitrary functions in $S$. Since $P_{e q}(x)>0$,

$$
\int_{\boldsymbol{R}^{n}} P_{e q}^{-1}(x) f(x) \mathcal{L}[g(x)] d x=\int_{\boldsymbol{R}^{n}} P_{e q}^{-1}(y) g(y) \mathcal{L}[f(y)] d y .
$$

Therefore, the operator $\mathcal{L}$ is symmetric with respect to the reciprocal of its stationary distribution $P_{e q}(x): w(x)=P_{e q}(x)$. This result is known to physicists.

(iv) $\Rightarrow(\mathbf{v})$. The differential operator $\mathcal{L}$ in $(22)$ can also be rewritten as

$$
\mathcal{L}[f]=\frac{1}{2} \nabla \cdot(A \nabla f)+(\nabla f) B x+\operatorname{Tr}[B] f
$$

where $\operatorname{Tr}[B]$ is the trace of the matrix $B$. The statement $(i v)$

$$
\int e^{U} g \mathcal{L}[f] d x=\int e^{U} f \mathcal{L}[g] d x
$$

in which the positive $w(x)=e^{-U}, f$ and $g \in S$ are arbitrary functions, leads to

$$
\int e^{U} g\left[\frac{1}{2} \nabla \cdot(A \nabla f)+(\nabla f) B x\right] d x=\int e^{U} f\left[\frac{1}{2} \nabla \cdot(A \nabla g)+(\nabla g) B x\right] d x .
$$

Through integration by parts, the first term on the left-hand-side (and similarly for the right-hand-side)

$$
\int e^{U} g \nabla \cdot(A \nabla f) d x=-\int e^{U}(\nabla g) A(\nabla f) d x-\int e^{U} g(\nabla U) A(\nabla f) d x,
$$

and we have

$$
\int e^{U} g\left[\frac{1}{2}(\nabla U) A(\nabla f)-(\nabla f) B x\right] d x=\int e^{U} f\left[\frac{1}{2}(\nabla U) A(\nabla g)-(\nabla g) B x\right] d x .
$$

By a simple rearrangement, we have

$$
\int e^{U}(g \nabla f-f \nabla g)\left(\frac{1}{2} A \nabla U-B x\right) d x=0 .
$$


Since $f$ and $g$ are arbitrary, we have $\frac{1}{2} A \nabla U-B x=0$ in which $U=-\log w$. Therefore

$$
\nabla \log w(x)+2 A^{-1} B x=0
$$

that is $e p r=0$.

(v) $\Rightarrow$ (i). In (9) $A$ is positive definite. Hence the integrand is always positive, therefore epr $=0$ implies $2 A^{-1} B x=-\nabla \log P_{e q}(x)$. Therefore $A^{-1} B$ is a symmetric matrix. Furthermore, $P_{e q}$ is a normal density and hence, $P_{e q} \in L^{1} \Rightarrow A^{-1} B$ is positive definite.

Corollary 3.1. A reversible stationary Gaussian solution to (11) has its covariance matrix $\Xi=\frac{1}{2} B^{-1} A$ where $B$ has to have all its eigenvalues being real and positive. It has a symmetric two-time covariant matrix $R\left(t, t^{\prime}\right)=e^{-B\left|t-t^{\prime}\right|} \Xi$, and its entropy production is zero.

\section{LINEAR THEORY FOR IRREVERSIBLE STATIONARY PROCESSES}

In the previous section, we have shown that a symmetric $A^{-1} B$ in (14) is a necessary and sufficient condition for reversibility of the stationary solution of (11). The result also implies that all the eigenvalues of matrix $B$ are necessarily positive. In this section, we consider the situation when such symmetric condition is absent. One interesting class of problems is when matrix $B$ has complex eigenvalues.

Definition 4.1. A linear stochastic system characterized by (11) and satisfies the statments in Theorem 3.1 is called reversible. A system which is not reversible is called irreversible.

Lemma 4.1. Fokker-Planck equation (14) with positive definite A but unrestricted $B$ has an unique fundamental solution. The solution is Gaussian with

$$
E[x(t)]=e^{-B t} x^{0}, \quad E\left[\Delta x(t) \Delta x(t)^{T}\right]=\int_{0}^{t} e^{-B s} A e^{-B^{T} s} d s, \quad(\forall t>0)(25)
$$

where $\Delta x(t)=x(t)-E[x(t)]$.

Proof. The proof relies on a direct verification of the Gaussian function with $(25)$ as a solution to equation (14). This has been done many times by physicists $[8,21]$. Hence we will not repeat the lengthy computation. With 
the verification of the solution, and uniqueness of the fundamental solution to (14), the lemma is proven.

THEOREM 4.1. The necessary and sufficient condition for nonsweeping is that matrix $B$ has all its eigenvalues with positive real parts.

Proof. Necessity: If the solution to (14) is nonsweeping, then by the Lemma 4.1 and the sweeping theorem from Section 1, its fundamental solution has a stationary limit $\in L^{1}$. Since $x(t)$ has Gaussian distribution for $t$, the limit distribution is also Gaussiani with finite variance. Therefore,

$$
\lim _{t \rightarrow \infty} E\left[\Delta x(t) \Delta x(t)^{T}\right]<\infty .
$$

By the general formula for $e^{-B s}[1]$ and using $A=\Gamma \Gamma^{T}$, the convergence of (26) implies that all the eigenvalues of $B$ in (25) must have positive real parts.

Sufficiency: If all the eigenvalues have positive real parts, the nonstationary Gaussian solution in (25) has a unique Gaussian density as its limit when $t \rightarrow \infty$. With the help of the fundamental solution for equation (14) and the Markovian property, a stationary Gaussian process related to the quantities in (25) can be constructed. Hence the solution of (14) are nonsweeping.

COROLlary 4.1. The necessary and sufficient condition for a stationary Gaussian process to be irreversible is the matrix $B$ in (11) has all eigenvalues with positive real parts and $A^{-1} B$ is nonsymmetric.

\section{A STRONG FORM OF FLUCTUATION-DISSIPATION RELATION}

We now limit our discussion to nonsweeping situation with and without reversibility. We first give

Definition 5.1. The matrix relation among $B, \Gamma$ in (11), and the stationary covariance $\Xi$ of $x(t)$

$$
\Gamma \Gamma^{T}=B \Xi+\Xi B^{T}
$$

is called standard fluctuation-dissipation relation. Actually,

$$
\Xi=\lim _{t \rightarrow \infty} \int_{0}^{t} e^{-B s} A e^{-B^{T} s} d s .
$$


Combining the above Theorem 3.1 and Corollary 4.1, we immediately have the following corollary.

COROLlary 5.1. The standard fluctuation-dissipation relation is a necessary but not sufficient condition for the reversibility of system (11). For a reversible system, it can be simplified into a stronger form

$$
A=2 B \Xi
$$

Proof. By Theorem 3.1, (11) with reversibility has symmetric $\Gamma^{-T} \Gamma^{-1} B$; and by Corollary 3.1 its stationary process has covariance $\Xi=\frac{1}{2} B^{-1} \Gamma \Gamma^{T}$. Therefore, $B \Xi=\frac{1}{2} \Gamma \Gamma^{T}$ is symmetric, and

$$
\Gamma \Gamma^{T}=2 B \Xi=B \Xi+\Xi B^{T} .
$$

Hence, the standard fluctuation-dissiplatin relation follows, and furthermore (28) can be simplified into $A=2 B \Xi$.

On the other hand, the standard fluctuation-dissipation relation is satisfied by any stationary Gaussian processes with or without the symmetric $A^{-1} B$ : From (25) we have

$$
B \int_{0}^{t} e^{-B s} A e^{-B^{T} s} d s+\left(\int_{0}^{t} e^{-B s} A e^{-B^{T} s} d s\right) B^{T}=-\left[e^{-B t} A e^{-B^{T} t}\right]_{0}^{t} .
$$

Let $t \rightarrow \infty$, the upper limit vanishes and we have

$$
B \Xi+\Xi B^{T}=A .
$$

Hence by Corollary 4.1 it is not a sufficient condition for reversibility.

(27) has a close resemblance to Einstein's original fluctuation-dissipation relation, in which $A$ is the covariance of the fluctuating white noise, $B$ is the dissipative linear relaxation rates, and $\Xi$ is the equilibrium covariance $(k T)$.

\section{THE ONSAGER'S HYPOTHESIS AND GREEN-KUBO FORMULA}

The following statement is known as Onsager's hypothesis or GreenKubo formula [10].

THEOREM 6.1. If a system in (11) is reversible, the conditional expectation $E\left[x(t) \mid x_{0}\right]$ and the two-time covariance of its stationary solution have 
identical time dependence in the following sense:

$$
E\left[x(t) \mid x_{0}\right]=e^{-B t} x_{0}, \quad R(t, 0)=e^{-B t} \Xi,
$$

where $\Xi=E\left[x x^{T}\right]$ is the covariance of the stationary solution.

Proof. The proof of this result is contained in the proof of Theorem 3.1, $(i) \Rightarrow(i i)$.

Remark: This is Onsager's original statement [10]. However, the statement is not limited to reversible systems, it is also applicable to nonsweeping irreversible systems:

$$
R(t, 0)=E_{x_{0}}\left[E\left[x \mid x_{0}\right] x_{0}^{T}\right]=E_{x_{0}}\left[e^{-B t} x_{0} x_{0}^{T}\right]=e^{-B t} \Xi .
$$

Indeed, this result which relies solely on the first equality in (29) is in fact a consequence of the linearity of equation (11).

\section{REFERENCES}

1. E. A. Coddington and N. Levinson, Theory of Ordinary Differential Equations, McGraw-Hill, New York, 1955.

2. R. R. Fox, Gaussian Stochastic Processes in Physics, Phys. Rep., 48 (1978), pp. 180283.

3. M. Z. Guo, M. QIAn, And Z. D. Wang, The Entropy Production and Circulation of Diffusion Processes on Manifold, Chin. Sci. Bull., 42 (1998), pp. 982-985.

4. T.L. Hill, Free Energy Transduction and Biochemical Cycle Kinetics, SpringerVerlag, New York, 1995.

5. F. Jülicher, A. Ajdari, And J. Prost, Modeling Molecular Motors, Rev. Mod. Phys., 69 (1997), pp 1269-1281.

6. A. Lasota And M. C. MaCkey, Chaos, Fractals, and Noise: Stochastic Aspects of Dynamics, Springer-Verlag, New York, 1994.

7. J.L. Lebowitz And H. Spohn, A Gallavotti-Cohen-Type Symmetry in the Large Deviation Functional for Stochastic Dynamics, J. Stat. Phys., 95 (2000), pp 333365 .

8. J. KeIzer, Statistical Thermodynamics of Nonequilibrium Processes, SpringerVerlag, New York, 1987.

9. B. K. ØKSEndal, Stochastic Differential Equations: An Introduction with Applications, third Ed., Springer-Verlag, New York, 1997.

10. L. Onsager, Reciprocal Relations in Irreversible Processes. I, Phys. Rev., 37 (1931), pp. 405-426.

11. H. QIAN, Vector Field Formalism and Analysis for a Class of Thermal Ratchets, Phys. Rev. Lett., 81 (1998), pp. 3063-3066.

12. H. QIAN, Single-Particle Tracking: Brownian Dynamics of Viscoelastic Materials, Biophys. J., 79 (2000), pp. 137-143. 
13. H. QIAN, A Mathematical Analysis for the Brownian Dynamics of DNA Tether, J. Math. Biol., 41 (2000), pp. 331-340.

14. H. QIAN, The Mathematical Theory of Molecular Motor Movement and Chemomechanical Energy Transduction, J. Math. Chem., in the press.

15. H. QIAN, Equations for Stochastic Macromolecular Mechanics of Single Proteins: Equilibrium Fluctuations, Transient Kinetics and Nonequilibrium Steady-State, J. Chem. Phys., submitted.

16. H. QIAN, Nonequilibrium Steady-State Circulations and Heat Dissipation Functional, Phys. Rev. E., in the press.

17. H. QIAN, Relative Entropy: Free Energy Associated with Equilibrium Fluctuations and Nonequilibrium Deviations, Phys. Rev. E., in the press.

18. M. QIAN AND Z. D. WAng, The Entropy Production of Diffusion Processes on Manifolds and Its Circulation Decomposition, Commum. Math. Phys., 206 (1999), pp. $429-445$.

19. M. P. QIAN AND M. QIAN, The Entropy Production and Irreversibility of Markov Processes, Chin. Sci. Bull., 30 (1985), pp. 445-447.

20. M. P. QIAN, M. QIAN, AND G. L. Gong, The Reversibility and the Entropy Production of Markov Processes, Contemp. Math., 118 (1991), pp. 255-261.

21. N. G. Van Kampen, Stochastic Processes in Physics and Chemistry, Revised and enlarged Ed., North-Holland, Amsterdam, 1997.

22. M. C. Wang And G. E. UhlenBeck, On the Theory of the Brownian Motion II, Rev. Mod. Phys., 17 (1945), pp. 323-342.

23. N. Wax, Selected Papers on Noise and Stochastic Processes, Dover, New York, 1954.

24. G. WeIss, Time-Reversibility of Linear Stochastic Processes, J. Appl. Prob., 12 (1975), pp. 831-836. 